\title{
Exotic Radionuclides - What are they good for?
}

\author{
Dorothea Schumann*, Emilio Maugeri, and Rugard Dressler
}

\begin{abstract}
Accelerator waste contains a number of rare isotopes that are urgently needed in various fields of scientific research. Activated components from the surroundings of high-power accelerators like the HIPA cyclotron at PSI are valuable sources for such 'isotope mining'. While the isotope production itself is practically 'for free', because the irradiation takes place anyway, the challenge is the chemical extraction of the required isotopes from the activated matrix material. The article presents an overview on the attempts and achievements of more than 15 years research and development in the research group 'Isotope and Target Chemistry' at PSI and presents some of the highlights in scientific applications.
\end{abstract}

Keywords: Geoscience · Isotope production · Nuclear astrophysics · Nuclear data · Radiochemical separation

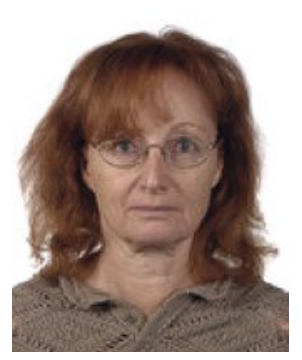

Dorothea Schumann studied chemistry at the Technical University (TU) in Dresden, Germany and obtained her $\mathrm{PhD}$ after five years of study at the Joint Institut of Nuclear Research in Dubna, Russia. Up to 1999 she held a position as scientist in the working group Radiochemistry in the Institut for Analytics at TU Dresden, where she studied the chemical properties of Superheavy Elements. After a short intermediate stop as a project manager in the company Wälischmiller she switched to Paul Scherrer Institut Villigen, Switzerland in 2000, where she has been working as group leader of the research group Isotope and Targetchemie since then. Her research interests focus on the production and separation of exotic radionuclides from accelerator waste, their radioanalytics as well as the determination of their decay data (half-lives, branching ratios) and reaction rates.

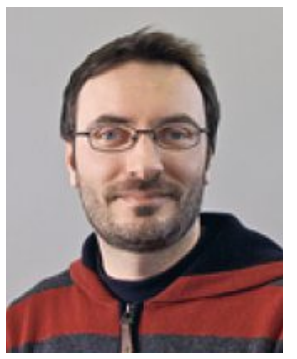

Emilio A. Maugeri got his $\mathrm{PhD}, 2009$, at the Institute for Transuranium Elements, Germany (issued by the University of Pavia, Italy), where he studied the microstructural aspects of the evolution of $\alpha$-radiation damage and helium behavior in spent nuclear fuel. After that he used his competence to develop a new project on helium behavior in glass matrices used to immobilize nuclear waste at the Commissariat à l'Énergie Atomique, Marcoule, France, where he worked as post-doc from 2010 to 2011. He has been working at Paul Scherrer Institute since 2012, first as a post-doc and after as a permanent staff member, on liquid metals, implementation radiochemical separation and targets preparation methods, for nuclear physics and nuclear astrophysics research.

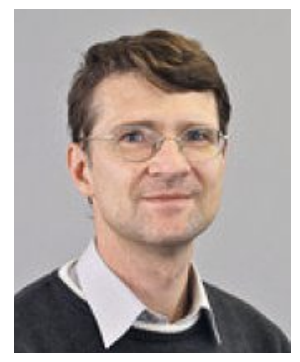

Rugard Dressler studied nuclear physics and theoretical physics at the Universities of Leipzig and Berlin, Germany. During his studies he began working closely with radiochemists to study the physical and chemical properties of the isotopes of the heaviest elements. In 1999, he received his $\mathrm{PhD}$ from the University of Bern, Switzerland, for reaction studies on the synthesis of nobelium, rutherfordium and dubnium isotopes. Since 2001 he has been working at the Paul Scherrer Institute in the group of 'heaviest elements' on the investigation of chemical properties of the transactinides from rutherfordium $(Z=104)$ to fleurovium $(Z=114)$. Furthermore, he conducted or contributed to experiments with astrophysically interesting isotopes such as ${ }^{7} \mathrm{Be},{ }^{44} \mathrm{Ti},{ }^{53} \mathrm{Mn}$ and ${ }^{60} \mathrm{Fe}$.

\section{Introduction}

When we talk about 'exotic radionuclides', we must first define what we mean. In the field of nuclear physics, the term 'exotic nuclei' is often used to characterize isotopes exhibiting unusual nuclear properties. Examples of such statements are: "Exotic nuclei are known to exhibit qualitatively new phenomena and provide a new testing ground for our understanding of quantum many-body science"[1] or: "Exotic Nuclei: These nuclei are highly unstable and lie very far from the valley of $\beta$-stability. Such nuclei exhibit unusual phenomena and provide an extreme test of models of nuclear structure". [2] These definitions focus on the nuclear properties of the isotopes and their potential application for solving fundamental problems in nuclear structure and quantum physics. In contrast to this definition, we use the term 'exotic radionuclide' for very difficult to obtain and therefore extremely rare isotopes, whose production consequently requires enormous efforts, whereas their nuclear properties are of secondary interest and the fields of application are not limited to basic nuclear physics.

The task is particularly challenging in cases where no stable neighboring isotopes exist and if no appropriate compound nuclear reactions are available, because neutron capture or evaporation will not result in the production of the desired isotopes.

The dedicated production of such relatively long-lived radionuclides can be both costly and time-consuming due to the required extremely long irradiation periods, and in some special cases, e.g. with ${ }^{60} \mathrm{Fe}$ or ${ }^{32} \mathrm{Si}$, it can make the production of suf-
*Correspondence: Dr. D. Schumann, E-mail: dorothea.schumann@psi.ch Paul Scherrer Institut Villigen, Forschungsstrasse 111, CH-5232 Villigen, Switzerland 
ficient quantities almost impossible. On the other hand, some of these isotopes are in high demand in several research areas. An effective solution to meet the experimental requirements could be the exploitation of waste from high-power, high-energy particle accelerators. Primary and secondary particles produced in such facilities induce in matter the production of a broad variety of isotopes; ranging from compound reaction nuclei with masses a few units higher than the target mass, down to the lightest nuclei produced by spallation and fragmentation reactions. The advantage of such 'isotope mining' consists in the principal presence of these isotopes; its production does not require 'extra' beam time. The challenge is their radiochemical isolation from the matrix. Since the primary source material consists of a mixture of a huge number of radionuclides, accompanied by the macro amount of the matrix, sophisticated and highly selective radiochemical separation methods have to be developed to obtain the required isotope in sufficient quantities with the desired radiochemical purity. Interestingly, although radiochemical methods have essentially improved since the first isolation of a significant amount of radium from pitchblende by Marie Curie in 1904, ${ }^{[3]}$ the relationship between the amount of source material and the weight of the finally isolated product has not changed essentially, as we will show below using some prominent examples.

To answer the title question "...what are they good for?", we need to look closer into Nature. The origin and development of the Universe and the chemical elements is one of the fundamental scientific questions of humankind. To gain deeper insight into the driving forces of galactic and stellar processes, one needs to study the corresponding nuclear reactions, which comprise highenergetic neutron and particle induced reactions, followed by all kinds of nuclear disintegration. To a certain extent, the environment in a high-energetic particle accelerator is similar to the situation during galactic evolution processes. Therefore, it is obvious that some of the radionuclides produced in the environment of such an accelerator play also a role in nuclear astrophysics. In particular, so-called 'waiting points' in element evolution appearing in the s-process during a supernova explosion, i.e. long-lived radionuclides allowing for further neutron capture before the normally following $\beta^{-}$decay occurs, can be found in considerable amounts also in accelerator waste. The most prominent example in this field is ${ }^{60} \mathrm{Fe}$, which we will discuss in more detail later on. On the other hand, light radioactive isotopes play a key role in the Big Bang Theory, one of them being ${ }^{7} \mathrm{Be}$, one of the parameters in the so-called 'Cosmological Lithium Problem (CLIP)', which we will address in Section 4.2. As the third example, we selected recently performed experiments with the radionuclide ${ }^{53} \mathrm{Mn}$, a cosmogenic radionuclide that decays by electron capture. It is of interest for geological research as a marker for age determination of rocks and sediments, for dating the formation and alterations in meteorites in early stages of our solar system, and for astrophysically relevant nuclear processes in supernova explosions. Finally, we give a short outlook on currently running projects and future challenges.

\section{Tasks for Radiochemists}

Exploring the nuclear data basis for 'exotic radionuclides', it becomes evident that, in a considerable number of cases, basic information on half-lives, branching ratios and cross sections is unreliable, insufficiently determined or missing. The reasons for this unsatisfying situation are manifold, the most important two being the limited amount of available sample material and, in some cases, the difficulties in the detection and quantification of their radiation (especially for so-called 'hard-to-measure-isotopes'). Consequently, we define the tasks for us as radiochemists as follows:

- Identification and analysis of source material for exotic radionuclides
- Development of separation procedures for extraction of the radionuclides from the matrix material

- Target and sample preparation and characterization, tailored for envisaged experiments

- Performance of scientific experiments in the field of cross section and half-life determination.

With this approach, we follow the vision of Marie Curie, expressed in her PhD thesis: "... we thought it well to hand over specimens of the substances, discovered and prepared by ourselves, to certain physicists, in the first place to $H$. Bequerel ... . In this way, we ourselves facilitated the research by others besides ourselves on the new radioactive bodies.'[3]

\section{Isotope Production Possibilities at PS}

PSI operates the Spallation Neutron Source SINQ, which is driven by one of the most powerful high-energetic proton accelerators worldwide: the High Intensity Proton Accelerators HIPA (590 MeV, up to $2.4 \mathrm{~mA}$ ), and is therefore best-suited as a producer of exotic radionuclides. In the framework of the ERAWAST (Exotic Radionuclides from Accelerator Waste for Science and Technology ${ }^{[4]}$ ) initiative, a complex program for isotope separation from different matrices has been established at PSI within the last 15 years.

Fig. 1 shows a schematic view of the present PSI accelerator facilities. The arrows point to special positions, from which useful material can be extracted.

Several of potential isotope sources like the ones shown in Fig. 1 were examined using extended analytical procedures in order to qualify them for possible exploitation regarding the preparation of exotic radionuclide samples. For example, we determined the radionuclide inventory and the spatial distribution of selected radionuclides for one of the lead targets of the SINQ, ${ }^{[5]}$ one of the carbon wheels from the muon production station ${ }^{[6]}$ and the copper beam dump. ${ }^{[6]}$ For the extraction of valuable sample material, dedicated separation procedures were developed, for instance for ${ }^{10} \mathrm{Be}$ from graphite, ${ }^{[8]}$ for ${ }^{44} \mathrm{Ti},{ }^{53} \mathrm{Mn}$ and ${ }^{26} \mathrm{Al}$ from STIP samples ${ }^{[9]}$ or ${ }^{7} \mathrm{Be}$ from the SINQ cooling water. ${ }^{[10]}$

\section{Selected Examples for Application of Exotic Radionuclides}

\section{$4.1{ }^{60} \mathrm{Fe}$ - 'Hot Spot' in Super Novae Explosion?}

A hype on the radioactive isotope ${ }^{60} \mathrm{Fe}$ was observed over the past 15 years in nuclear astrophysics research. After Knie et al. ${ }^{[11]}$ reported around 20 years ago on the first observation of hints for a nearby supernovae (SN) explosion in terrestrial archives, several independent experimental studies have been published subsequently. One of the scientific instruments installed on the Advanced Composition Explorer (ACE), the mass spectrometer CRIS, recorded ${ }^{60} \mathrm{Fe}$ in galactic cosmic rays. ${ }^{[12]}$ Moreover, ${ }^{60} \mathrm{Fe}$ was detected and quantified in ocean floor samples, ${ }^{[13]}$ on the surface of the moon, ${ }^{[14]}$ in Earth's microfossil records ${ }^{[15]}$ and meteorites. ${ }^{[16]}$ These findings are accompanied by theoretical works, confirming the experimental results. ${ }^{[17]}$ The most sensational showpiece, however, is the recently published identification of ${ }^{60} \mathrm{Fe}$ and ${ }^{53} \mathrm{Mn}$ in fresh Antarctic snow samples, ${ }^{[18]}$ referring to evidence for a recent change in the ${ }^{60} \mathrm{Fe}$ influx to Earth from the solar neighborhood environment.

With this high scientific relevance for the entire astrophysics research in the mass region around iron, all complementary studies related to ${ }^{60} \mathrm{Fe}$, in particular the re-determination of the halflife as well as neutron capture cross-section measurements are of similarly high impact, because such nuclear data are the basis for the evaluation and interpretation of the recent findings.

Unfortunately, ${ }^{60} \mathrm{Fe}$ is very rare in geological and meteoritic samples, and the artificial production is extremely difficult. With two mass units heavier than the heaviest stable iron isotope, ${ }^{58} \mathrm{Fe}$, 


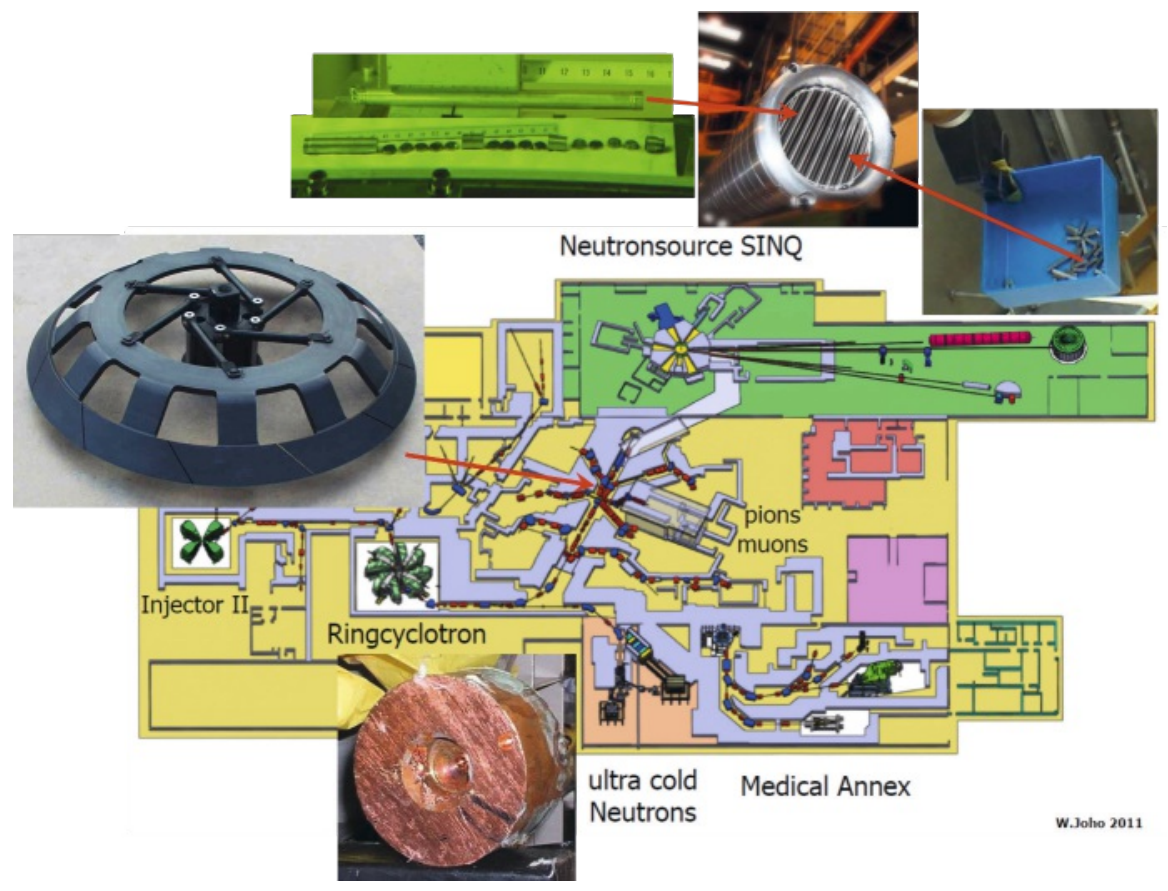

Fig. 1. Schematic view of the PSI accelerator complex with some sources for exotic isotopes. (courtesy of Werner Joho). The inset images show a SINQ target with examined $\mathrm{Pb}$-target rods and STIP specimens (top), a carbon wheel for muon production (middle) and a part of the copper beam-dump of the former BMA (bottom).

it is barely accessible by direct neutron activation (only via double neutron capture with very low cross section) and only in extremely limited yield by compound nuclei reactions with charged particles (no suitable isotopes of the needed beam and target elements are available). The only feasible option is spallation, induced by highenergetic particles on target elements with a higher atomic number than iron. A copper beam-dump (Fig. 1 and Fig. 2), coming from the former Bio-Medical-Area BMA at PSI and dismounted in 1992, which had been irradiated over a time span of more than 10 years with high-energetic protons from the HIPA, is one of the few suitable sources worldwide for significant quantities of this isotope. After identifying the areas with the highest isotope concentration within the 10-kg-heavy copper block (Fig. 2 right), ${ }^{[7]}$ the radionuclide-bearing spots were drilled out and underwent several radiochemical processes.

Around $10^{16}$ atoms of ${ }^{60} \mathrm{Fe}$, corresponding to around $1 \mu \mathrm{g}$ out of $3 \mathrm{~g}$ copper could be separated from the matrix and accompanying other isotopes, e.g. ${ }^{60} \mathrm{Co}$ as the main contaminant as well as ${ }^{26} \mathrm{Al}$ and ${ }^{53} \mathrm{Mn}$, which are interesting for other scientific experiments (for ${ }^{53} \mathrm{Mn}$, see Section 4.3). The finally obtained ${ }^{60} \mathrm{Fe}$ batch was transformed into samples for several dedicated experiments, in particular for three new half-life determinations ${ }^{[19]}$ and two neutron capture cross-section measurements. ${ }^{[20]}$ The three half-life measurements consistently yielded a more than one million years higher value with essentially reduced uncertainty, instead of the formerly known 1.5 Mio years, thus requiring a re-evaluation of the models applied so far. The neutron capture cross sections both for thermal and stellar energies ${ }^{[20]}$ were the first-ever measurements for this isotope, with which now a much more reliable experimental database exists for the interpretation of related astrophysical processes.

Moreover, we manufactured and characterized a series of reference materials for Accelerator Mass Spectrometry (AMS), ${ }^{[21]}$ a very sensitive detection method, which is nowadays able to determine isotopic ratios down to ratios of $10^{-14}$, in special cases even lower. However, AMS is not an absolute method, but performs the measurements in comparison to reference material with wellknown isotopic ratios. Due to the extreme rareness, no reference material for ${ }^{60} \mathrm{Fe}$ was available so far. With these novel standards, the accurateness and uncertainties of results obtained by AMS can be improved significantly and results from former measurements, e.g. see refs $[14,15,18]$, can be re-scaled, once the direct cross check of the formerly used material with the new reference material had been performed at the corresponding AMS facility.

Conclusion: Yes, ${ }^{60} \mathrm{Fe}$ is definitely one of the hot topics in nuclear astrophysics. With our sample material, we could essentially contribute to the improvement of the nuclear database necessary to better understand processes of star evolution and element synthesis in the mass region around iron.
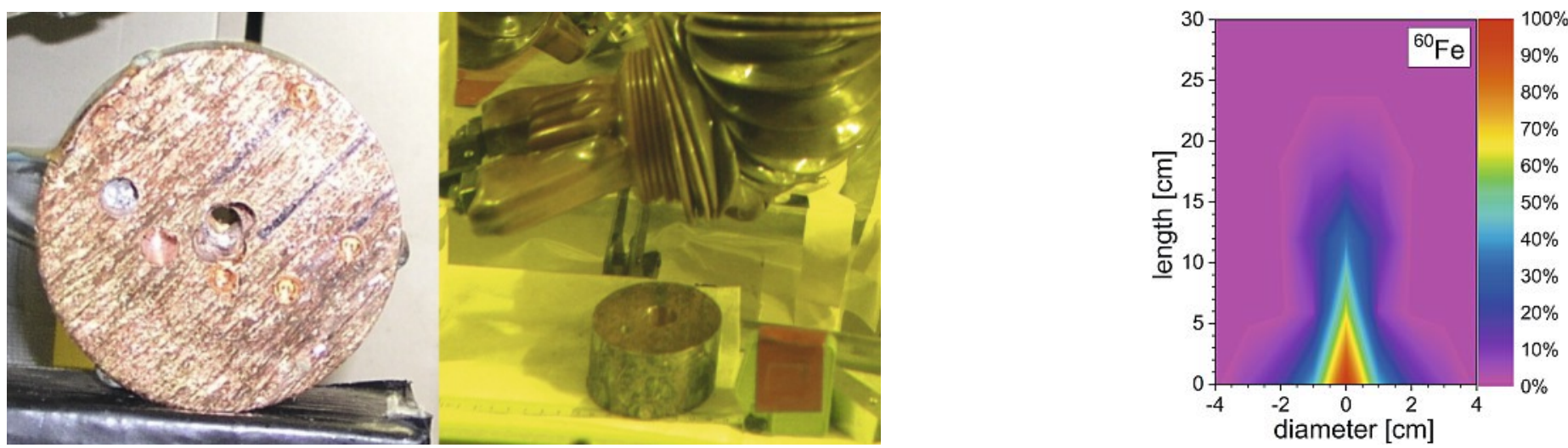

Fig. 2. Left: Photo of the copper beam dump, segment 3, where the sample-taking positions are shown. Right: Segment after drilling; note the much bigger hole in the middle of the segment. The mechanical work had to be performed in a hotcell in the Hotlab of PSI, due to the high dose rate caused by ${ }^{60} \mathrm{Co}$. The right-hand picture shows the relative distribution of ${ }^{60} \mathrm{Fe}$ inside the beam-dump. 


\section{2 ${ }^{7} \mathrm{Be}$ - Solution for an Unsolved Puzzle in Big Bang Theory?}

One of the most long lasting and controversial problems in nuclear astrophysics, the so-called 'Cosmological Lithium Problem' (CLiP) consists in a discrepancy of a factor 3-4 between the primordial ${ }^{7} \mathrm{Li}$ abundance as calculated using the standard Big-Bang nucleosynthesis model (BBN model $)^{[22]}$ and once measured in the atmosphere of metal-poor stars in the Galactic halo. ${ }^{[23]}$ This inconsistency has undermined the validity of the BBN model itself, which, on the other hand, has perfectly predicted the abundance of the other light isotopes produced during the BBN in the early Universe (specifically, within the first few minutes after the Big Bang), such as ${ }^{4} \mathrm{He},{ }^{3} \mathrm{He},{ }^{3} \mathrm{H}$ and ${ }^{2} \mathrm{H}$. About $90 \%$ of the primordial ${ }^{7} \mathrm{Li}$ has radiogenic origin, deriving from the electron capture of ${ }^{7} \mathrm{Be}$, which was also produced in the early Universe. ${ }^{[24]}$ Thus, to solve the CLiP, it was necessary to have an accurate knowledge of the nuclear reaction cross sections leading to the production and destruction of ${ }^{7} \mathrm{Be}$ during this phase of the Universe. The cross section of the main channels for the production and destruction of ${ }^{7} \mathrm{Be}$, i.e. ${ }^{3} \mathrm{He}(\alpha, \gamma){ }^{7} \mathrm{Be} e^{[25]}$ and ${ }^{7} \mathrm{Be}(\mathrm{n}, \mathrm{p})^{7} \mathrm{Li}$, respectively, have been known with good precision since the late 1980s.[26] However, a direct measurement of the ${ }^{7} \mathrm{Be}(\mathrm{n}, \mathrm{p})^{7} \mathrm{Li}$ cross-sections in the BBNregion, $10-100 \mathrm{keV}$ and, especially, of the ${ }^{7} \mathrm{Be}(\mathrm{n}, \alpha)^{4} \mathrm{He}$ cross section, which is the second most important destruction channel, had been lacking until few years ago, with exception of the thermal capture cross section. The problem that had hindered the implementation of these measurements was related to the use of several $\mu \mathrm{g}$ of ${ }^{7} \mathrm{Be}\left(\mathrm{t}_{1 / 2}=53.29 \mathrm{~d}\right)$ as target material, which is difficult both to obtain with high chemical purity and to handle because of its very high specific activity $(13 \mathrm{GBq} / \mu \mathrm{g})$.

${ }^{7} \mathrm{Be}$ can be extracted at PSI from the cooling water of the SINQ facility, where it is produced in large amounts by spallation reactions on ${ }^{16} \mathrm{O}$, installing a filter with an ion-exchange resin $\left(\right.$ LEWATIT $\left.^{\circledR}\right)$ into one of the SINQ cooling water loops. ${ }^{[10]}$ After the necessary radiochemical separation and purification steps, which have to be performed remote-controlled in a hotcell (see Fig. 3), several hundred GBq of ${ }^{7} \mathrm{Be}$ were obtained, enabling the manufacture of a number of requested targets for several planned astrophysics experiments.

Two targets were produced, ${ }^{[27]}$ applying molecular plating and droplet deposition, for the first direct measurement campaign of the $\left.{ }^{7} \mathrm{Be}(\mathrm{n}, \alpha)\right)^{4} \mathrm{He}$ cross section from $10 \mathrm{meV}$ to $10 \mathrm{keV}$ neutron energy, performed at the n_TOF-CERN facility (Geneva, Switzerland). [28] To measure the ${ }^{7} \mathrm{Be}(\mathrm{n}, \mathrm{p})^{7} \mathrm{Li}$ cross-section directly, an isotopically

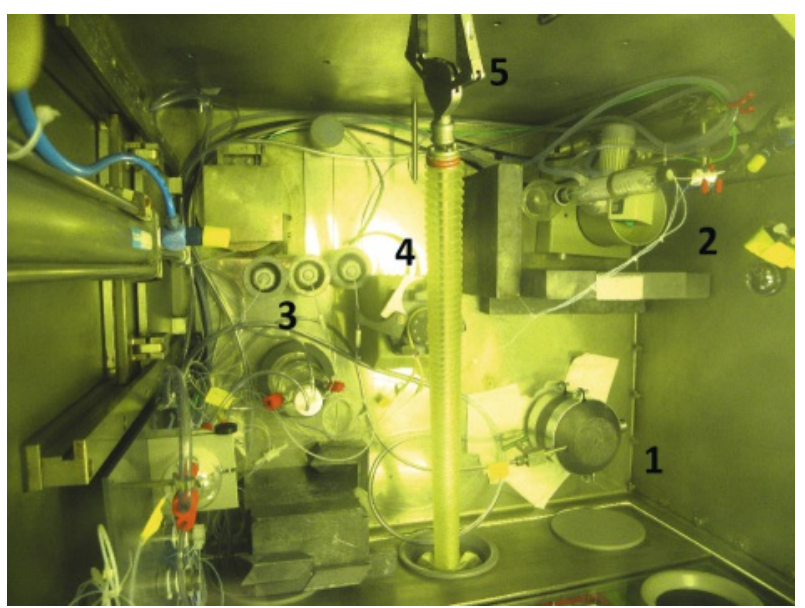

Fig. 3. Photo of the remote-controlled radiochemical separation setup, installed in a lead-shielded hot-cell. The photo was taken from the ceiling and shows the shielded filter vessel (1), the vacuum evaporator (2), the collection vessels for the final products (3), the pump (4), several transfer tubes, and, the long stick in the middle, the manipulator (5), which allows interaction from outside when the hotcell is locked. pure ${ }^{7} \mathrm{Be}$ target was produced at the ISOLDE-CERN facility by implanting the isotope into thin aluminum disks ${ }^{[29]}$ using another ${ }^{7} \mathrm{Be}$ batch from PSI. This target allowed a very precise measurement of the ${ }^{7} \mathrm{Be}(\mathrm{n}, \mathrm{p})^{7} \mathrm{Li}$ cross section at neutron energies up to $300 \mathrm{keV} .^{[30]}$ Although both of these cross-section measurements resulted in values with high precision and low uncertainty, the results did not explain the deviation of around a factor 3-4 and, thus, did not solve the CLIP. Complementary experiments, also using implanted targets, were performed at the reactors at ILL (Grenoble, France) and Rez (Czech Republic), in order to determine the neutron capture cross sections at thermal and cold neutron energies. [31]

Conclusion: No, neutron capture reactions on ${ }^{7} \mathrm{Be}$ do not solve the 'Cosmological Lithium problem'. Nevertheless, with the help of our sample material, these reactions could be ruled out as possible reasons for the presently existing discrepancies. The recently obtained results using our sample material essentially improved the nuclear database for neutron-induced reactions in the region of light masses.

\section{3 ${ }^{53} \mathrm{Mn}$ - Stepchild in Nuclear Astrophysics and Geoscience?}

${ }^{53} \mathrm{Mn}$ is a cosmogenic radionuclide synthesized in freeze out phases of explosive burning in supernovae explosions. ${ }^{[32]}$ Furthermore, it can also be produced by spallation reactions with high-energy cosmic rays (mainly protons or neutrons) on isotopes in the iron region both in meteorites and on Earth. The second production pathway can be used to date processes at the earth's surface within the last 15 million years by measuring the introduced ${ }^{53} \mathrm{Mn}$ concentration in terrestrial mass rock. Such processes at the Earth's surface may include changes in glacier covering during the last ice ages. ${ }^{[33]}$ Calculations of the galactic chemical evolution in our Milky Way showed ${ }^{53} \mathrm{Mn}$ being the most abundant short-lived radioisotope produced in thermonuclear and core collapsed supernovae. ${ }^{[34]}$ The amount of ${ }^{53} \mathrm{Mn}$ that will be introduced into the solar system after a supernova explosion and, thus, finally will be deposited on Earth, exceeds by more than one order of magnitude the steady-state deposition due to the production by high-energy cosmic rays as a kind of ambient background. ${ }^{[35]}$ Signatures of such supernovae events, which must have occurred at a distance of less than 160 light years away from our Sun, have already been found for ${ }^{60} \mathrm{Fe}$ in natural archives ${ }^{[17]}$ as discussed above.

Due to its 'hard-to-measure' radiation as a weak electron capture nuclide, ${ }^{53} \mathrm{Mn}$ cannot be observed directly at its astrophysical production site in contrast to other isotopes also produced during supernovae explosions such as ${ }^{26} \mathrm{Al},{ }^{44} \mathrm{Ti}$ or even ${ }^{60} \mathrm{Fe}$, which are easily detectable in space thanks to the $\gamma$-rays directly emitted by them or emitted by their daughter nuclei. This is one of the reasons why, compared to the immense interest ${ }^{60} \mathrm{Fe}$ has attracted in the field of nuclear astrophysics, ${ }^{53} \mathrm{Mn}$ has been given little attention so far. A possible second reason for the lack of data on ${ }^{53} \mathrm{Mn}$ is the extreme rareness of the isotope on Earth. The few measurements performed in the 1970 s to determine the neutron capture cross section at thermal energy ${ }^{[36]}$ and the half-life ${ }^{[36 a, 37]}$ were performed with sample amounts of $10^{13}$ atoms only, extracted in extremely laborious work from meteorite material. However, with the development of the elaborated detection techniques, ${ }^{53} \mathrm{Mn}$ could, similar to ${ }^{60} \mathrm{Fe}$, recently be detected in galactic cosmic rays by the CRIS spectrometer ${ }^{[12]}$ or determined in form of 'cosmic fallout' of condensed interstellar material in sediments on Earth's surface. Traces of ${ }^{53} \mathrm{Mn}$, together with ${ }^{60} \mathrm{Fe}$, were found in Antarctic snow, ${ }^{[18]}$ as mentioned above. Such accreted interstellar dust can help to identify and date past supernovae events occurring in the stellar neighborhood of our solar system. ${ }^{[17 a, 38]}$ The interest in ${ }^{53} \mathrm{Mn}$ has been rising in the last years as the technical improvements in the performance of several AMS facilities allowed reliable detection of ${ }^{53} \mathrm{Mn}$ in terrestrial materials with 
very low detection limits. This could enable the utilization of the isotope as a tool for geological dating of environmental samples and even could help to explain some discrepancies discussed in the literature when comparing data of the ${ }^{53} \mathrm{Mn} /{ }^{53} \mathrm{Cr}$ chronometer with other chronometers in meteorites originating from the early phases of the Solar system, if its half-life would have been known with acceptable precision and uncertainty.

Despite the lack of half-life measurements, also the data on neutron capture cross sections for ${ }^{53} \mathrm{Mn}$ are scarce. Besides the thermal neutron capture cross section, no experimental data were available until recently. Experimentally determined cross sections in the keV range are, however, necessary to understand stellar evolution and nucleosynthesis.

In the framework of the PhD thesis of J. Ulrich, ${ }^{[39]}$ a complex program was elaborated to measure all missing neutron capture cross section data in the range from very cold to stellar neutron energies at different facilities worldwide, using the activation technique. An overview of these measurements is shown in Fig. 4.

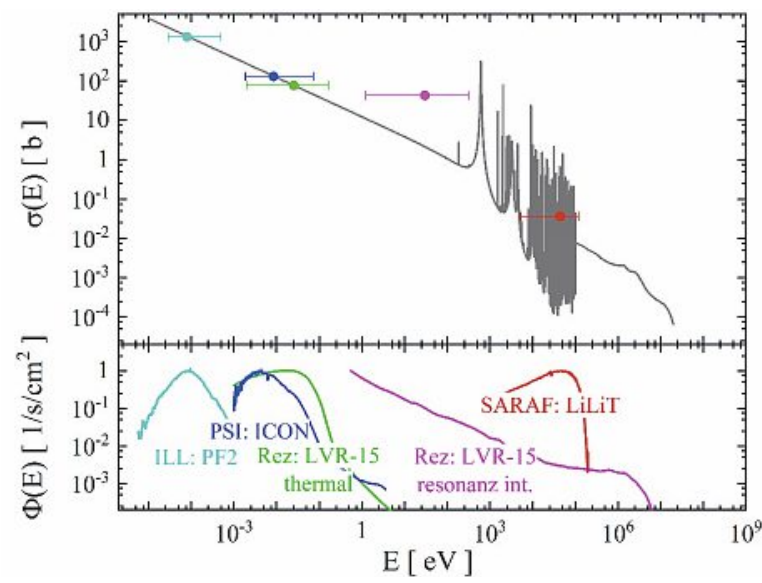

Fig. 4. Neutron capture cross section measurements of ${ }^{53} \mathrm{Mn}$ performed in the frame of the PhD work of J. Ulrich. The top panel displays the capture cross sections (colored points) measured at various facilities, together with the energy interval covering $90 \%$ of applied neutron flux indicated as error bars of the energy axis. The uncertainty of the cross sections is below $3 \%$ ( $95 \%$ confidence interval) about a third of the size of the gray line, which represents TALYS cross section predictions (https://tendl.web.psi.ch/tendl_2019/tendl2019.html). The position and strength of the resonances were tuned to reproduce the experimental data, especially the resonance integral and MACS. The lower panel shows the normalized neutron spectra of the used experimental facilities. The colors correspond to those in the upper panel.

For the preparation of the required ${ }^{53} \mathrm{Mn}$ samples, we used two different sources: Copper chips from the beam dump of the former Bio-Medical-Area at PSI (see Fig. 1 and Section 4.1) and samples from the so-called STIP program, ${ }^{[40]}$ a material research program at PSI aimed to investigate mechanical property changes in structure material during high dose irradiations. Several stainless steel samples were used after the corresponding stress test investigations for extracting ${ }^{44} \mathrm{Ti},{ }^{26} \mathrm{Al}$ and ${ }^{53} \mathrm{Mn} \cdot{ }^{[9,41]}$ From both sources, we manufactured batches containing up to $10^{19}$ atoms of ${ }^{53} \mathrm{Mn}$, this amounting to around five orders of magnitude more than ever available for experiments world-wide.

As visible in Fig. 4, the results for the thermal neutron cross section, measured at the research reactor LVR-15 in Rez (Czech Republic), and for the cold neutrons using the ICON beamline at PSI, reproduce well the values known from literature[36a] with an essentially improved uncertainty. ${ }^{[42]}$ Moreover, it was possible for the first time, using the LiLiT source at SOREQ (Israel), to obtain a Maxwellian Averaged Cross Section (MACS), matching stellar neutron energies of $20-50 \mathrm{keV} \cdot{ }^{[43]}$ The work is completed by measuring the interaction of very cold neutrons at ILL with ${ }^{53} \mathrm{Mn}$.

In parallel, a re-determination of the ${ }^{53} \mathrm{Mn}$ half-life was performed in order to utilize the isotope as a dating tool for geological and environmental samples. For this purpose, a mass separation had to be performed at the University of Mainz to produce an isotopically pure sample for the activity measurement of the 'hardto-measure' deexcitation radiation of ${ }^{53} \mathrm{Mn}$. The obtained value for the ${ }^{53} \mathrm{Mn}$ half-life is slightly lower than the literature reference, but was, again, determined with a much-improved uncertainty of less than $2 \%$.[39]

Conclusion: No, due to recent improvements in the detection techniques and thanks to our results on cross section and halflife measurements as well as the newly provided AMS reference material, ${ }^{53} \mathrm{Mn}$ will no longer be considered a wallflower in the nuclear astrophysics landscape, but has the potential to develop into a powerful tool for geological, environmental and astrophysics research.

\subsection{Samples from Isotopes not Produced at PSI}

Finally, we want to mention, that we are also able to process material that does not originate from PSI facilities, for instance samples irradiated at the high-flux reactor at ILL (Grenoble, France) or the cyclotron of the Inselspital (Berne, Switzerland). Examples for such successful sample preparation and target manufacturing from 'outside samples', used in dedicated scientific experiments, are e.g. the neutron capture cross section measurements of ${ }^{63} \mathrm{Ni},{ }^{[44]}{ }^{171} \mathrm{Tm}$ and ${ }^{147} \mathrm{Pm}$ at n_TOF (CERN, Switzerland) and SARAF (SOREQ, Israel), respectively, ${ }^{[45,48]}$ as well as the determination of the atomic transitions and the first ionization potential of promethium at University Mainz (Germany).[46]

\section{Conclusions and Outlook}

After launching the first ERAWAST initiative at PSI in 2006 with an ESF-funded workshop at PSI, ${ }^{[4]}$ we have continuously been gaining experience on isotope extraction and sample/target preparation for a broad variety of scientific applications. As a result, the Isotope and Target Chemistry Group at PSI owns nowadays a worldwide unique stock of 'exotic radionuclides'. Besides the isotopes ${ }^{60} \mathrm{Fe},{ }^{53} \mathrm{Mn}$ and ${ }^{7} \mathrm{Be}$ discussed here, we have samples of ${ }^{44} \mathrm{Ti},{ }^{26} \mathrm{Al},{ }^{10} \mathrm{Be}$ and many others available. Currently, we are working on the separation of several long-lived lanthanides $\left({ }^{146} \mathrm{Sm},{ }^{148 / 152} \mathrm{Gd},{ }^{154} \mathrm{Dy},{ }^{157 / 158} \mathrm{~Tb},{ }^{137} \mathrm{La}\right)$ from irradiated Ta for halflive determinations ${ }^{[47]}$ as well as ${ }^{32} \mathrm{Si}$ from irradiated $\mathrm{V}$, foreseen to be utilized as nuclear dating tool for environmental samples. We provide the material for interesting, challenging and exiting scientific experiments all over the world, in a broad field of research and applications. A remarkable number of well-recognized results has been published in high-ranking scientific journals, for instance in Physical Review Letters: six have already appeared, [19a,b,20a,28,30,44] one is accepted ${ }^{[48]}$ and one is currently under revision. ${ }^{[43]}$ As a special highlight, it has to be emphasized, that, so far, all experiments on nuclear properties of ${ }^{60} \mathrm{Fe}$ using macro amounts, such as half-life and neutron capture cross sections within the last 20 years, were conducted with samples in which the material was produced and the samples were tailor-made at PSI.

The application fields for our 'exotic radionuclides' are manifold; they not only cover geological, environmental, astrophysics, medical or nuclear energy research, but also contribute to technical improvements of state-of-the-art measurement techniques like AMS or Liquid Scintillation Counting. However, we are not only able to produce and provide sample and target material for experiments governed by other groups, but - thanks to the work performed with ${ }^{53} \mathrm{Mn}$ - we could convincingly demonstrate that our group can design, rule and successfully perform our own experiments on half-life and cross section measurements. The currently running projects on long-lived lanthanides and ${ }^{32} \mathrm{Si}$, both 
funded by the Swiss National Science Foundation (SNSF), will continue this promising approach to establish the radiochemistry laboratory at PSI as an internationally acknowledged expert team for isotope and target production and related nuclear data measurements.

To further improve our impact in the research fields described here and in the future, we are currently exploring the possibilities to design and build a dedicated isotope separator at PSI for exotic isotopes. This would give us the chance to provide isotopically pure samples, which are urgently needed in several important fields of nuclear research. An EC-funded project (SANDA) provides the basis to perform a first design study, which hopefully is followed later on by the installation of the facility in the Hotlab at PSI. This device, together with the radiochemical expertise, will give the radiochemistry at PSI a world-wide leadership position for isotope and target production and related research.

\section{Acknowledgements}

A huge number of colleagues, present and former colleagues from PSI as well as the collaborators, listed as co-authors in the cited publications, contributed essentially to the work outlined in this article. We want to express our thanks to all of them. Special thanks go to M. Ayranov, T. Wieseler and S. Heinitz for their outstanding laboratory work. The projects were partially funded by the European Commission in the frame of FP7 and HORIZON2020.

Received: July 28, 2020

[1] R. F. Casten, B. M. Sherrill, Prog. Part. Nucl. Phys. 2000, 45, S171, https://doi.org/10.1016/S0146-6410(00)90013-9.

[2] https://www.supa.ac.uk/Research/NPP/NP_Physics_EN.html.

[3] M. Curie, PhD Thesis La Faculté des Sciences de Paris, Paris, 1903.

[4] https://www.psi.ch/de/lrc/erawast.

[5] a) T. Lorenz, PhD thesis University of Berne, Switzerland, 2015; b) T. Lorenz, Y. Dai, D. Schumann, Radiochim. Acta 2013, 101, 661, https://doi.org/10.1524/ract.2013.2067; c) T. Lorenz, Y. Dai, D. Schumann, A. Türler, Nuclear Data Sheets 2014, 119, 284, https://doi.org/10.1016/j.nds.2014.08.078.

[6] a) D. Schumann, S. Lüthi, T. Stowasser, D. Kiselev, S. Teichmann, in 'Radiocarbon', University of Arizona, Tucson, Arizona 2012, p. 370; b) D. Schumann, J. Neuhausen, S. Horn, P. W. Kubik, I. Günther-Leopold, Radiochim. Acta 2008, 96, 31, https://doi.org/10.1524/ract.2008.1462

[7] D. Schumann, J. Neuhausen, J. Eikenberg, M. Ruthi, M. Wohlmuther, P. W. Kubik, H. A. Synal, V. Alfimov, G. Korschinek, G. Rugel, T. Faestermann, Radiochim. Acta 2009, 97, 123, https://doi.org/10.1524/ract.2009.1585.

[8] S. Heinitz, D. Kiselev, N. Kivel, D. Schumann, Appl. Radiat. Isotopes 2017, 130, 260, https://doi.org/10.1016/j.apradiso.2017.10.012.

[9] R. Dressler, M. Ayranov, D. Bemmerer, M. Bunka, Y. Dai, C. Lederer, J. Fallis, A. S. Murphy, M. Pignatari, D. Schumann, T. Stora, T. Stowasser, F. K. Thielemann, P. J. Woods, J. Phys. G: Nucl. Part. Phys. 2012, 39, https://doi.org/10.1088/0954-3899/39/10/105201

[10] D. Schumann, M. Ayranov, T. Stowasser, L. Gialanella, A. di Leva, M. Romano, D. Schuermann, Radiochim. Acta 2013, 101, 509, https://doi.org/10.1524/ract.2013.2078.

[11] a) K. Knie, S. Merchel, G. Korschinek, T. Faestermann, U. Herpers, M. Gloris, R. Michel, Meteoritics \& Planetary Sci. 1999, 34, 729, https://doi.org/10.1111/j.1945-5100.1999.tb01385.x; b) K. Knie, G. Korschinek, T. Faestermann, E. A. Dorfi, G. Rugel, A. Wallner, Phys. Rev. Lett. 2004, 93, https://doi.org/10.1103/PhysRevLett.93.171103.

[12] W. R. Binns, M. H. Israel, E. R. Christian, A. C. Cummings, G. A. de Nolfo, K. A. Lave, R. A. Leske, R. A. Mewaldt, E. C. Stone, T. T. von Rosenvinge, M. E. Wiedenbeck, Science 2016, 352, 677, https://doi.org/10.1126/science.aad6004.

[13] A. Wallner, J. Feige, N. Kinoshita, M. Paul, L. K. Fifield, R. Golser, M. Honda, U. Linnemann, H. Matsuzaki, S. Merchel, G. Rugel, S. G. Tims, P. Steier, T. Yamagata, S. R. Winkler, Nature 2016, 532, 69, https://doi.org/10.1038/nature17196.

[14] L. Fimiani, D. L. Cook, T. Faestermann, J. M. Gomez-Guzman, K. Hain, G. Herzog, K. Knie, G. Korschinek, P. Ludwig, J. Park, R. C. Reedy, G. Rugel, Phys. Rev. Lett. 2016, 116, https://doi.org/10.1103/PhysRevLett.116.151104.

[15] P. Ludwig, S. Bishop, R. Egli, V. Chernenko, B. Deneva, T. Faestermann, N. Famulok, L. Fimiani, J. M. Gomez-Guzman, K. Hain, G. Korschinek, M. Hanzlik, S. Merchel, G. Rugel, Proc. Natl. Acad. Sci. U. S. A. 2016, 113, 9232, https://doi.org/10.1073/pnas.1601040113.

[16] I. Leya, J. C. David, T. Faestermann, M. Froehlich, N. Kivel, D. Koll, G. Korschinek, S. McIntyre, S. Merchel, S. Pavetich, G. Rugel, D. Schumann,
T. Smith, A. Wallner, Meteoritics \& Planetary Sci. 2020, 55, 818, https://doi.org/10.1111/maps.13466.

[17] a) B. J. Fry, B. D. Fields, J. R. Ellis, Astrophys. J. 2016, 827, https://doi.org/10.3847/0004-637x/827/1/48; b) D. Breitschwerdt, J. Feige, M. M. Schulreich, M. A. d. Avillez, C. Dettbarn, B. Fuchs, Nature 2016, 532, 73, https://doi.org/10.1038/nature17424.

[18] D. Koll, G. Korschinek, T. Faestermann, J. M. Gomez-Guzman, S. Kipfstuhl, S. Merchel, J. M. Welch, Phys. Rev. Lett. 2019, 123, https://doi.org/10.1103/PhysRevLett.123.072701.

[19] a) G. Rugel, T. Faestermann, K. Knie, G. Korschinek, M. Poutivtsev, D. Schumann, N. Kivel, I. Gunther-Leopold, R. Weinreich, M. Wohlmuther, Phys. Rev. Lett. 2009, 103, https://doi.org/10.1103/PhysRevLett.103.072502; b) A. Wallner, M. Bichler, K. Buczak, R. Dressler, L. K. Fifield, D. Schumann, J. H. Sterba, S. G. Tims, G. Wallner, W. Kutschera, Phys. Rev. Lett. 2015, 114, https://doi.org/10.1103/PhysRevLett.114.041101; c) K M. Ostdiek, T. S. Anderson, W. K. Bauder, M. R. Bowers, A. M. Clark, P. Collon, W. Lu, A. D. Nelson, D. Robertson, M. Skulski, R. Dressler, D. Schumann, J. P. Greene, W. Kutschera, M. Paul, Phys. Rev. C 2017, 95 https://doi.org/10.1103/PhysRevC.95.055809.

[20] a) E. Uberseder, R. Reifarth, D. Schumann, I. Dillmann, C. D. Pardo, J. Görres, M. Heil, F. Käppeler, J. Marganiec, J. Neuhausen, M. Pignatari, F. Voss, S. Walter, M. Wiescher, Phys. Rev. Lett. 2009, 102, 151101, https://doi.org/10.1103/PhysRevLett.102.151101; b) T. Heftrich, M. Bichler, R. Dressler, K. Eberhardt, A. Endres, J. Glorius, K. Gobel, G. Hampel, M. Heftrich, F. Kappeler, C. Lederer, M. Mikorski, R. Plag, R. Reifarth, C. Stieghorst, S. Schmidt, D. Schumann, Z. Slavkovska, K. Sonnabend, A. Wallner, M. Weigand, N. Wiehl, S. Zauner, Phys. Rev. C 2015, 92, https://doi.org/10.1103/PhysRevC.92.015806.

[21] D. Schumann, N. Kivel, R. Dressler, Plos One 2019, 14 https://doi.org/10.1371/journal.pone.0219039.

[22] G. Steigman, Annu. Rev. Nucl. Part. Sci. 2007, 57, 463 , https://doi.org/10.1146/annurev.nucl.56.080805.140437.

[23] a) F. Spite, M. Spite, Astronomy Astrophys. 1982, 115, 357; b) M. Asplund, D. L. Lambert, P. E. Nissen, F. Primas, V. V. Smith, Astrophys. J. 2006, 644, 229, https://doi.org/10.1086/503538.

[24] R. Khatri, R. A. Sunyaev, Astronomy Lett. 2011, 37, 367, https://doi.org/10.1134/s1063773711060041

[25] G. Gyürky, F. Confortola, H. Costantini, A. Formicola, D. Bemmerer, R. Bonetti, C. Broggini, P. Corvisiero, Z. Elekes, Z. Fülöp, G. Gervino, A Guglielmetti, C. Gustavino, G. Imbriani, M. Junker, M. Laubenstein, A. Lemut, B. Limata, V. Lozza, M. Marta, R. Menegazzo, P. Prati, V. Roca, C. Rolfs, C. R. Alvarez, E. Somorjai, O. Straniero, F. Strieder, F. Terrasi, H. P. Trautvetter (LUNA Collaboration), Phys. Rev. C 2007, 75, 035805 , https://doi.org/10.1103/PhysRevC.75.035805.

[26] a) Y. M. Gledenov, T. S. Zvarova, I. Kvitek, M. P. Mitrikov, R. S. Mitrikova, Y. P. Popov, V. I. Salatsky, F. Van Zuan, in 'International Conference on Neutron Physics', Kiev, 1987, p. 232; b) P. E. Koehler, C. D. Bowman, F. J. Steinkruger, D. C. Moody, G. M. Hale, J. W. Starner, S. A. Wender, R. C. Haight, P. W. Lisowski, W. L. Talbert, Phys. Rev. C 1988, 37, 917, https://doi.org/10.1103/PhysRevC.37.917.

[27] E. A. Maugeri, S. Heinitz, R. Dressler, M. Barbagallo, N. Kivel, D. Schumann, M. Ayranov, A. Musumarra, M. Gai, N. Colonna, M. Paul, S. Halfon, L. Cosentino, P. Finocchiaro, A. Pappalardo, JINST 2017, 12, P02016.

[28] M. Barbagallo, et al. (n_TOF Collaboration), Phys. Rev. Lett. 2016, 117, 152701

[29] E. A. Maugeri, S. Heinitz, R. Dressler, M. Barbagallo, J. Ulrich, D. Schumann, N. Colonna, U. Köster, M. Ayranov, P. Vontobel, M. Mastromarco, J. Schell, J. M. Correia, T. Stora, Nucl. Instrum. Methods Phys. Res., Sect. A 2018 889, 138, https://doi.org/https://doi.org/10.1016/j.nima.2018.01.078.

[30] L. Damone, M. Barbagallo, M. Mastromarco, A. Mengoni, L. Cosentino, E. Maugeri, S. Heinitz, D. Schumann, R. Dressler, F. Käppeler, N. Colonna, P. Finocchiaro, J. Andrzejewski, J. Perkowski, A. Gawlik, O. Aberle, S. Altstadt, M. Ayranov, L. Audouin, M. Bacak, J. Balibrea-Correa, J. Ballof, V. Bécares, F. Bečvár, C. Beinrucker, G. Bellia, A. P. Bernardes, E. Berthoumieux, J. Billowes, M. J. G. Borge, D. Bosnar, A. Brown, M. Brugger, M. Busso, M. Caamaño, F. Calviño, M. Calviani, D. Cano-Ott, R. Cardella, A. Casanovas, D. M. Castelluccio, R. Catherall, F. Cerutti, Y H. Chen, E. Chiaveri, J. G. M. Correia, G. Cortés, M. A. Cortés-Giraldo, S. Cristallo, M. Diakaki, M. Dietz, C. Domingo-Pardo, A. Dorsival, E. Dupont, I. Duran, B. Fernandez-Dominguez, A. Ferrari, P. Ferreira, W. Furman, S. Ganesan, A. García-Rios, S. Gilardoni, T. Glodariu, K. Göbel, I. F. Gonçalves, E. González-Romero, T. D. Goodacre, E. Griesmayer, C. Guerrero, F. Gunsing, H. Harada, T. Heftrich, J. Heyse, D. G. Jenkins, E. Jericha, K. Johnston, Y. Kadi, A. Kalamara, T. Katabuchi, P. Kavrigin, A. Kimura, N. Kivel, U. Köster, M. Kokkoris, M. Krtička, D. Kurtulgil, E. LealCidoncha, C. Lederer-Woods, H. Leeb, J. Lerendegui-Marco, S. Lo Meo, S J. Lonsdale, R. Losito, D. Macina, J. Marganiec, B. Marsh, T. Martínez, A. Masi, C. Massimi, P. Mastinu, F. Matteucci, A. Mazzone, E. Mendoza, P. M. Milazzo, F. Mingrone, M. Mirea, A. Musumarra, A. Negret, R. Nolte, A Oprea, N. Patronis, A. Pavlik, L. Piersanti, M. Piscopo, A. Plompen, I. Porras, J. Praena, J. M. Quesada, D. Radeck, K. Rajeev, T. Rauscher, R. Reifarth, 
A. Riego-Perez, S. Rothe, P. Rout, C. Rubbia, J. Ryan, M. Sabaté-Gilarte, A. Saxena, J. Schell, P. Schillebeeckx, S. Schmidt, P. Sedyshev, C. Seiffert, A. G. Smith, N. V. Sosnin, A. Stamatopoulos, T. Stora, G. Tagliente, J. L. Tain, A. Tarifeño-Saldivia, L. Tassan-Got, A. Tsinganis, S. Valenta, G. Vannini, V. Variale, P. Vaz, A. Ventura, V. Vlachoudis, R. Vlastou, A. Wallner, S. Warren, M. Weigand, C. Weiß, C. Wolf, P. J. Woods, T. Wright, P. Žugec, Phys. Rev. Lett. 2018, 121, 042701, https://doi.org/10.1103/PhysRevLett.121.042701.

[31] I. Tomandl, J. Vacik, U. Koster, L. Viererbl, E. A. Maugeri, S. Heinitz, D. Schumann, M. Ayranov, J. Ballof, R. Catherall, K. Chrysalidis, T. D. Goodacre, D. Fedorov, V. Fedosseev, K. Johnston, B. Marsh, S. Rothe, J. Schell, C. Seiffert, Phys. Rev. C 2019, 99, https://doi.org/10.1103/PhysRevC.99.014612.

[32] a) F. K. Thielemann, K. Nomoto, M. Hashimoto, Astrophys. J. 1996, 460, 408, https://doi.org/10.1086/176980; b) K. Maeda, F. K. Ropke, M. Fink, W. Hillebrandt, C. Travaglio, F. K. Thielemann, Astrophys. J. 2010, 712, 624, https://doi.org/10.1088/0004-637x/712/1/624.

[33] J. M. Schaefer, T. Faestermann, G. F. Herzog, K. Knie, G. Korschinek, J. Masarik, A. Meier, M. Poutivtsev, G. Rugel, C. Schlüchter, F. Serifiddin, G. Winckler, Earth. Planet. Sci. Lett. 2006, 251, 334, https://doi.org/10.1016/j.eps1.2006.09.016.

[34] S. Sahijpal, J. Astrophys. Astronomy 2013, 34, 297, https://doi.org/10.1007/s12036-013-9188-2.

[35] B. D. Fields, K. A. Hochmuth, J. Ellis, Astrophys. J. 2005, 621, 902, https://doi.org/10.1086/427797.

[36] a) R. Wölfele, W. Herr, U. Herpers, Radiochim. Acta 1972, 18, 207, https://doi.org/10.1524/ract.1972.18.4.207; b) U. Herpers, R. Wölfle, W. Herr, J. Radioanal. Chem. 1973, 18, 145, https://doi.org/10.1007/BF02520698.

[37] M. Honda, M. Imamura, Phys. Rev. C 1971, 4, 1182 , https://doi.org/10.1103/PhysRevC.4.1182.

[38] a) J. Feige, A. Wallner, S. R. Winkler, S. Merchel, L. K. Fifield, G. Korschinek, G. Rugel, D. Breitschwerdt, Publ. Astronomical Soc. Australia 2012, 29, 109, https://doi.org/10.1071/AS11070; b) J. Feige, A. Wallner, L. K. Fifield, G. Korschinek, S. Merchel, G. Rugel, P. Steier, S. R. Winkler, R. Golser, EPJ Web of Conferences 2013, 63, 03003.

[39] J. Ulrich, PhD Thesis Berne, University Berne, 2020.

[40] Y. Dai, G. S. Bauer, J. Nucl. Mater. 2001, 296, 43, https://doi.org/10.1016/S0022-3115(01)00544-X.

[41] a) D. Schumann, T. Stowasser, R. Dressler, M. Ayranov, Radiochim. Acta 2013, 101, 501, https://doi.org/10.1524/ract.2013.2058; b) D. Schumann, M. Ayranov, J. Phys.: Conf. Ser. 2010, 202, 012034, https://doi.org/10.1088/1742-6596/202/1/012034.

[42] J. Ulrich, M. Ayranov, A. Kaestner, D. Schumann, P. Sprung, A. Türler, L. Viererbl, M. Vin, H. Assmann Vratislavská, R. Dressler, Phys. Rev. C 2020, accepted.

[43] J. Ulrich, M. Ayranov, O. Aviv, A. Barak, D. Berkovits, Y. Buzaglo, H. Dafna, B. Kaizer, N. Kivel, D. Kijel, A. Kreisel, M. Paul, E. Peretz, D. Rochman, D. Schumann, P. Sprung, M. Tessler, A. Türler, L. Weissman, Z. Yungrais, R. Dressler, Phys. Rev. Lett. 2020, in revision.

[44] C. Lederer et al., n TOF Collaboration, Phys. Rev. Lett. 2013, 110, https://doi.org/10.1103/PhysRevLett.110.022501.
[45] a) S. Heinitz, E. A. Maugeri, D. Schumann, R. Dressler, N. Kivel, C. Guerrero, U. Koster, M. Tessler, M. Paul, S. Halfon, Radiochim. Acta 2017, 105, 801, https://doi.org/10.1515/ract-2016-2728; b) C. Guerrero, M. Tessler, M. Paul, J. Lerendegui-Marco, S. Heinitz, E. A. Maugeri, C Domingo-Pardo, R. Dressler, S. Halfon, N. Kivel, U. Koster, T. PalchanHazan, J. M. Quesada, D. Schumann, L. Weissman, Phys. Lett. B 2019, 797, https://doi.org/10.1016/j.physletb.2019.134809.

[46] D. Studer, J. Ulrich, S. Braccini, T. S. Carzaniga, R. Dressler, K. Eberhardt, R. Heinke, U. Köster, S. Raeder, K. Wendt, Eur. Phys. J. A 2020, 56, 69 , https://doi.org/10.1140/epja/s10050-020-00061-8.

[47] N. M. Chiera, Z. Talip, A. Fankhauser, D. Schumann, PLOS ONE 2020, 15, e0235711, https://doi.org/10.1371/journal.pone.0235711.

[48] C. Guerrero, J. Lerendegui-Marco, M. Paul, M. Tessler, S. Heinitz, C. Domingo-Pardo, S. Cristallo, R. Dressler, S. Halfon, N. Kivel, U. Köster, E. A. Maugeri, T. Palchan-Hazan, J. M. Quesada, D. Rochman, D. Schumann, L. Weissman, O. Aberle, S. Amaducci, J. Andrzejewski, L. Audouin, V. Bécares, M. Bacak, J. Balibrea, A. Barak, M. Barbagallo, S. Barros, F. Bečvář, C. Beinrucker, D. Berkovits, E. Berthoumieux, J. Billowes, D. Bosnar, M. Brugger, Y. Buzaglo, M. Caamaño, F. Calviño, M. Calviani, D. Cano-Ott, R. Cardella, A. Casanovas, D. M. Castelluccio, F. Cerutti, Y. H. Chen, E. Chiaveri, N. Colonna, G. Cortés, M. A. Cortés-Giraldo, L. Cosentino, H. Dafna, A. Damone, M. Diakaki, M. Dietz, E. Dupont, I. Durán, Y. Eisen, B. Fernández-Domínguez, A. Ferrari, P. Ferreira, P. Finocchiaro, V Furman, K. Göbel, A. R. García, A. Gawlik, T. Glodariu, I. F. Gonçalves, E. González-Romero, A. Goverdovski, E. Griesmayer, F. Gunsing, H. Harada, T. Heftrich, J. Heyse, T. Hirsh, D. G. Jenkins, E. Jericha, F. Käppeler, Y. Kadi, B. Kaizer, T. Katabuchi, P. Kavrigin, V. Ketlerov, V. Khryachkov, D. Kijel, A. Kimura, M. Kokkoris, A. Kriese, M. Krtička, E. Leal-Cidoncha, C. LedererWoods, H. Leeb, S. Lo Meo, S. J. Lonsdale, R. Losito, D. Macina, A. Manna, J. Marganiec, T. Martínez, C. Massimi, P. Mastinu, M. Mastromarco, F. Matteucci, E. Mendoza, A. Mengoni, P. M. Milazzo, M. A. Millán-Callado, F. Mingrone, M. Mirea, S. Montesano, A. Musumarra, R. Nolte, A. Oprea, N. Patronis, A. Pavlik, J. Perkowski, L. Piersanti, I. Porras, J. Praena1, K. Rajeev, T. Rauscher, R. Reifarth, T. Rodríguez-González, P. C. Rout, C. Rubbia, J. A Ryan, M. Sabaté-Gilarte, A. Saxena, P. Schillebeeckx, S. Schmidt, A. Shor, P. Sedyshev, A. G. Smith, A. Stamatopoulos, G. Tagliente, J. L. Tain6, A. Tarifeño-Saldivia, L. Tassan-Got, A. Tsinganis, S. Valenta, G. Vannini, V. Variale, P. Vaz, A. Ventura, V. Vlachoudis, R. Vlastou, A. Wallner, S. Warren, M. Weigand, C. Weiss, C. Wolf, P. J. Woods, T. Wright, P. Žugec, Phys. Rev. Lett. 2020, 125, 142701, https://doi.org/10.1103/PhysRevLett.125.142701.

\section{License and Terms}

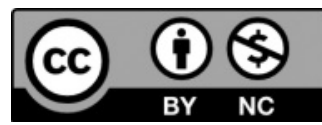

This is an Open Access article under the terms of the Creative Commons Attribution License CC BY_NC 4.0. The material may not be used for commercial purposes.

The license is subject to the CHIMIA terms and conditions: (http:// chimia.ch/component/sppagebuilder/?view=page\&id=12).

The definitive version of this article is the electronic one that can be found at https://doi.org/10.2533/chimia.2020.932 